\title{
EFFECTS OF DUST EXTINCTION IN GALACTIC DISCS
}

\author{
A. DI BARTOLOMEO ${ }^{1}$, G. BARBARO ${ }^{1}$ AND M. PERINOTTO ${ }^{2}$ \\ ${ }^{1}$ Dip. di Astronomia, Università di Padova, Padova, Italy \\ ${ }^{2}$ Dip. di Astronomia, Università di Firenze, Firenze, Italy
}

We have solved the radiative transfer equation taking into account both absorption and scattering into the line of sight to the observer to model the disc of spiral galaxies.

A dust model has been adopted, suitable for the diffuse interstellar medium of our Galaxy, to obtain, in all the considered spectral range $(1000 \div 10000 \AA)$, consistent quantities to describe both the absorption and the scattering properties of the dust.

New extinction curves, showing the internal absorption in a spiral galaxy, both if the scaleheight of stars and dust is the same (case $A$ ) or different (case $B$ ), have been derived (?). The most important results are the following:

1. The neglect of the scattering source is a hard approximation. In fact at $\tau_{V}=0.72$ the overestimate of the internal absorption is of the order of $40 \%$ when the scaleheights of stars and dust are the same, and about $25 \div 30 \%$ if the scaleheights are different. Moreover it increases with the optical depth.

2. The scattering deeply changes the shape of the observed mean interstellar galactic extinction curve. For instance the UV-bump, at $\lambda=2200$ $\AA$ is lower and larger; the slope in the optical region is different.

3. The behaviour of the colour excesses, for cases $A$ and $B$, as function of the optical depth, is different.

4. The use of the $C$ parameter defined in studies of the surface brightness of differently inclined spirals, to infer the optical thickness is misleading, unless the $C$ values are $<0.45$. For $\tau>2 \div 3$ in case $A, C$ becomes negative.

\section{References}

Di Bartolomeo, A., Barbaro, G. \& Perinotto, M., MNRAS, 1995, accepted 